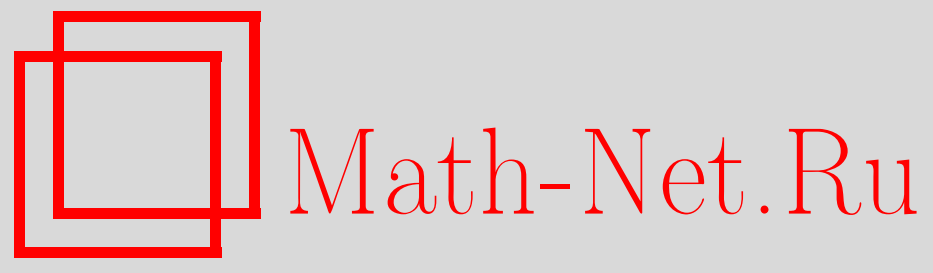

А. Б. Бейлин, Задача о колебаниях упруго закрепленного нагруженного стержня, Вестн. Caм. гос. техн. ун-та. Сер. Физ.-мат. науки, 2016, номер 2, 249-258

DOI: https://doi.org/10.14498/vsgtu1474

Использование Общероссийского математического портала MathNet.Ru подразумевает, что вы прочитали и согласны с пользовательским соглашением

http://www . mathnet.ru/rus/agreement

Параметры загрузки:

IP : 54.209 .52 .79

26 апреля 2023 г., 15:13:42

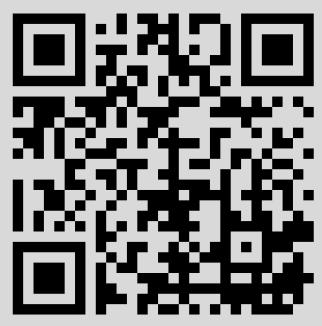


УДК 517.956.3

\title{
ЗАДАЧА О ПРОДОЛЬНЫХ КОЛЕБАНИЯХ УПРУГО ЗАКРЕПЛЕННОГО НАГРУЖЕННОГО СТЕРЖНЯ
}

\section{А. Б. Бейлин}

Самарский государственный технический университет, Россия, 443100, Самара, ул. Молодогвардейская, 244.

\begin{abstract}
Аннотация
Рассматриваются одномерные продольные колебания толстого короткого стержня, закреплённого на концах при помощи сосредоточенных масс и пружин. В качестве математической модели используется начально-краевая задача с динамическими краевыми условиями для гиперболического уравнения четвёртого порядка. Выбор именно этой модели обусловлен необходимостью учитывать эффекты деформации стержня в поперечном направлении, пренебрежение которыми, как показано Рэлеем, приводит к ошибке, что подтверждено современной нелокальной концепцией изучения колебаний твёрдых тел. Доказано существование ортогональной с нагрузкой системы собственных функций исследуемой задачи и получено их представление. Установленные свойства собственных функций позволили применить метод разделения переменных и доказать существование единственного решения поставленной задачи.
\end{abstract}

Ключевые слова: динамические краевые условия, продольные колебания, ортогональность с нагрузкой, модель Рэлея.

Введение. В любой работающей механической системе возникают колебательные процессы, которые могут порождаться различными причинами. Колебательные процессы могут быть следствием конструктивных особенностей системы или перераспределения нагрузок между различными элементами штатно работающей конструкции.

Наличие в механизме источников колебательных процессов может затруднить диагностику его состояния и даже привести к нарушению режима его работы, а в некоторых случаях и к разрушению. Различные проблемы, связанные с нарушением точности и работоспособности механических систем в результате вибрации некоторых их элементов, на практике часто решаются экспериментально.

Вместе с тем колебательные процессы могут быть весьма полезными, например, для обработки материалов, сборки и разборки соединений $[1$, с. 3, 4]. Ультразвуковые колебания позволяют не только интенсифицировать процессы резания (сверления, фрезерования, шлифования и т. д.) материалов с высокой твёрдостью (вольфрамосодержащих, титанокарбидных сталей и т. п.),

(C) 2016 Самарский государственный технический университет.

\section{Образец для цитирования}

Бейлин А. Б. Задача о продольных колебаниях упруго закрепленного нагруженного стержня // Вестн. Сам. гос. техн. ун-та. Сер. Физ.-мат. науки, 2016. Т. 20, № 2. С. 249258. doi: $10.14498 /$ vsgtu1474.

\section{Сведения об авторе}

Александр Борисович Бейлин (к.т.н, доц.; abeilin@mail.ru), доцент, каф. автоматизированных станочных и инструментальных систем. 
но в некоторых случаях стать единственно возможным методом обработки хрупких материалов (германий, кремний, стекло и т. д.) [2, с. 3]. Элемент устройства (волновод), который передаёт ультразвуковые колебания от источника (вибратора) до инструмента, называется концентратором и может иметь различную форму: цилиндрическую, коническую, ступенчатую, экспоненциальную и т. д. [3, с. 174]. Его предназначение - донести до инструмента колебания нужной амплитуды.

Таким образом, следствия протекания колебательных процессов могут быть различными, как и причины, их вызывающие, поэтому естественно возникает необходимость теоретического изучения процессов колебания. Математическая модель распространения волн в относительно длинных и тонких твёрдых стержнях, в основе которой лежит волновое уравнение второго порядка, хорошо изучена и давно стала классикой [4]. Однако, как показано Рэлеем [5, с. 273, 274], эта модель не вполне соответствует исследованию колебаний толстого короткого стержня, тогда как многие детали реальных механизмов можно интерпретировать как короткие и толстые стержни. В этом случае следует учитывать деформации стержня и в поперечном направлении. Математическая модель продольных колебаний толстого короткого стержня, в которой учтены эффекты поперечного движения стержня, называется стержнем Рэлея и базируется на гиперболическом уравнении четвёртого порядка [6, рр. 158-184]

$$
q(x) \frac{\partial^{2} u}{\partial t^{2}}-\frac{\partial}{\partial x}\left(a(x) \frac{\partial u}{\partial x}\right)-\frac{\partial}{\partial x}\left(b(x) \frac{\partial^{3} u}{\partial t^{2} \partial x}\right)=f(x, t)
$$

коэффициенты которого имеют физический смысл [7]:

$$
q(x)=\rho(x) A(x), \quad a(x)=A(x) E(x), \quad b(x)=\rho(x) \nu^{2}(x) I_{p}(x),
$$

где $A(x)$ - площадь поперечного сечения, $\rho(x)$ - массовая плотность стержня, $E(x)$ - модуль Юнга, $\nu(x)$ - коэффициент Пуассона, $I_{p}(x)$ - полярный момент инерции, $u(x, t)$ - продольные смещения, подлежащие определению.

Идеи Рэлея нашли своё подтверждение и развитие в современных работах, посвященных процессам колебаний, а также теории пластичности. В обзорной статье [8] обоснованы недостатки классических моделей, описывающих состояние и поведение твёрдых тел при нагрузке, в которых априори тело считается идеальным континуумом. Современный уровень развития естествознания требует построения новых моделей, адекватно описывающих исследуемые процессы, а разработанные в последние несколько десятилетий математические методы дают эту возможность. На этом пути в последнюю четверть прошлого века был предложен новый подход к изучению многих физических процессов, в том числе и упомянутых выше, основанный на понятии нелокальности (см. статью [8] и список литературы в ней). Один из классов нелокальных моделей, выделенных авторами, назван «слабо нелокальными». Математические модели, принадлежащие этому классу, могут быть реализованы введением в уравнение, описывающее некоторый процесс, производных высокого порядка, позволяющих учитывать в некотором приближении взаимодействие внутренних элементов объекта изучения. Таким образом, модель Рэлея актуальна и в наше время. 
1. Постановка задачи. Пусть концы стержня $x=0, x=l$ прикреплены к неподвижному основанию при помощи сосредоточенных масс $M_{1}, M_{2}$ и пружин, жёсткости которых $K_{1}$ и $K_{2}$. Будем считать, что стержень представляет собой тело вращения относительно оси $0 x$ и в начальный момент времени находится в покое в положении равновесия. Тогда мы приходим к следующей начально-краевой задаче.

ЗАдАчА. Найти в области $Q_{T}=\{(0, l) \times(0, T): l, T<\infty\}$ решение уравнения (1), удовлетворяющее начальным данным

$$
u(x, 0)=\varphi(x), \quad u_{t}(x, 0)=\psi(x)
$$

и граничным условиям

$$
\begin{aligned}
& a(0) u_{x}(0, t)+b(0) u_{x t t}(0, t)-K_{1} u(0, t)-M_{1} u_{t t}(0, t)=0, \\
& a(l) u_{x}(l, t)+b(l) u_{x t t}(l, t)+K_{2} u(l, t)+M_{2} u_{t t}(l, t)=0 .
\end{aligned}
$$

В статье [7] рассмотрены некоторые частные случаи задачи (1)-(2) и приведены примеры, в которых коэффициенты уравнения имеют явный вид и $M_{1}=M_{2}=0$. В статье [9] доказана однозначная слабая разрешимость поставленной задачи в общем случае.

Условия (2) обусловлены способом закрепления стержня: его концы прикреплены к неподвижным основаниям с помощью некоторых приспособлений, имеющих массы $M_{1}, M_{2}$, и пружин с жёсткостями $K_{1}, K_{2}$ соответственно. Наличие масс и учёт поперечных смещений приводит к условиям вида (2), содержащим производные по времени. Краевые условия, в которые входят производные по времени, называются динамическими. Они могут возникать в различных ситуациях, простейшие из которых описаны в учебнике [4], а гораздо более сложные - в монографии [10].

2. Изучение собственных колебаний стержня. Рассмотрим однородное уравнение, соответствующее уравнению (1). Так как коэффициенты зависят только от $x$, можно разделить переменные, представив $u(x, t)=X(x) T(t)$. Получим два уравнения:

$$
\begin{gathered}
T^{\prime \prime}(t)+\lambda^{2} T(t)=0 \\
\left(\left(a(x)-\lambda^{2} b(x)\right) X^{\prime}(x)\right)^{\prime}+\lambda^{2} q X(x)=0 .
\end{gathered}
$$

Уравнение (3) сопровождается краевыми условиями

$$
\begin{aligned}
& \left(a(0)-\lambda^{2} b(0)\right) X^{\prime}(0)-\left(K_{1}-\lambda^{2} M_{1}\right) X(0)=0, \\
& \left(a(l)-\lambda^{2} b(l)\right) X^{\prime}(l)+\left(K_{2}-\lambda^{2} M_{2}\right) X(l)=0 .
\end{aligned}
$$

Таким образом, мы пришли к задаче Штурма-Лиувилля, которая отличается от классической тем, что спектральный параметр $\lambda$ входит в коэффициент при старшей производной уравнения, а также в краевые условия. Это обстоятельство не позволяет ссылаться на известные из литературы результаты, поэтому нашей ближайшей целью является изучение задачи (3), (4). Для успешной реализации метода разделения переменных нам нужна информация о существовании и расположении собственных чисел, о качественных 
свойствах собственных функций: обладают ли они свойством ортогональности?

Покажем, что $\lambda^{2}>0$. Предположим, что это не так. Пусть $X(x)-$ собственная функция задачи (3), (4), соответствующая значению $\lambda=0$. Умножим (3) на $X(x)$ и проинтегрируем полученное равенство по промежутку $(0, l)$. Интегрируя по частям и применяя краевые условия (4), после элементарных преобразований получим

$$
\begin{aligned}
& \lambda^{2}\left[\left(a(0)-\lambda^{2} b(0)\right)\left(a(l)-\lambda^{2} b(l)\right) \int_{0}^{l}\left(q X^{2}+b X^{\prime 2}\right) d x+\right. \\
& \left.\quad+M_{1} X^{2}(0)+M_{2} X^{2}(l)\right]=\int_{0}^{l} a X^{\prime 2} d x+K_{1} X^{2}(0)+K_{2} X^{2}(l) .
\end{aligned}
$$

Заметим, что из физического смысла функции $a(x), b(x), q(x)$ положительны, $K_{i}, M_{i}$ неотрицательны. Но тогда из полученного равенства следует, что $X^{\prime}(x)=0, X(0)=X(l)=0$, следовательно, $X(x)=0$, что противоречит сделанному предположению. Стало быть, и предположение о том, что нуль есть собственное число задачи (3), (4) неверно.

Представление решения уравнения (3) зависит от знака выражения $a(x)-$ $-\lambda^{2} b(x)$. Покажем, что $a(x)-\lambda^{2} b(x)>0 \forall x \in(0, l)$. Зафиксируем произвольно $x \in(0, l)$ и найдём значения в этой точке функций $a(x), b(x), q(x)$. Запишем уравнение (3) в виде

$$
X^{\prime \prime}(x)+\mu X(x)=0
$$

где мы обозначили

$$
\mu=\frac{\lambda^{2} q}{a-\lambda^{2} b}
$$

в выбранной фиксированной точке, а условия (4) запишем в виде

$$
X^{\prime}(0)-\alpha X(0)=0, \quad X^{\prime}(l)+\beta X(l)=0,
$$

где $\alpha, \beta$ легко вычисляются.

Как известно, классическая задача Штурма-Лиувилля (5), (6) имеет счётное множество собственных функций при $\mu>0$, откуда в силу произвольности $x$ следует нужное неравенство.

Собственные функции задачи (3), (4) обладают свойством ортогональности с нагрузкой [4, с. 158], выраженным соотношением

$$
\begin{aligned}
\int_{0}^{l}\left(q X_{m}(x) X_{n}(x)+b X_{m}^{\prime}(x) X_{n}^{\prime}(x)\right) d x+ & \\
& +M_{1} X_{m}(0) X_{n}(0)+M_{2} X_{m}(l) X_{n}(l)=0,
\end{aligned}
$$

которое можно получить стандартным способом (см., например, [4, с. 159]), реализация которого в случае рассматриваемой задачи связана с элементарными, но кропотливыми вычислениями. Приведём кратко его вывод, опустив аргумент функций $X_{i}(x)$ во избежание громоздкости. 
Пусть $\lambda_{m}, \lambda_{n}$ - различные собственные числа, $X_{m}, X_{n}$ - соответствующие им собственные функции задачи (3), (4). Тогда

$$
\left(\left(a-\lambda_{m}^{2} b\right) X_{m}^{\prime}\right)^{\prime}+\lambda_{m}^{2} q X_{m}=0, \quad\left(\left(a-\lambda_{n}^{2} b\right) X_{n}^{\prime}\right)^{\prime}+\lambda_{n}^{2} q X_{n}=0 .
$$

Умножим первое из этих уравнений на $X_{n}$, а второе на $X_{m}$ и вычтем из первого второе. После элементарных преобразований получим равенство

$$
\left(\lambda_{m}^{2}-\lambda_{n}^{2}\right) q X_{m} X_{n}=\left(a X_{m} X_{n}^{\prime}\right)^{\prime}-\lambda_{n}^{2}\left(b X_{m} X_{n}^{\prime}\right)^{\prime}-\left(a X_{m}^{\prime} X_{n}\right)^{\prime}+\lambda_{m}^{2}\left(b X_{m}^{\prime} X_{n}\right)^{\prime}
$$

которое проинтегрируем по промежутку $(0, l)$. В результате, учитывая (4) и сокращая на $\left(\lambda_{m}^{2}-\lambda_{n}^{2}\right)$, получим соотношение $(7)$.

Доказанные утверждения о свойствах собственных чисел и собственных функций задачи Штурма-Лиувилля (3), (4) позволяют применить для отыскания решения поставленной задачи метод разделения переменных.

3. Разрешимость задачи. Обозначим

$$
\mathscr{C}\left(Q_{T}\right)=\left\{u: u \in C\left(\bar{Q}_{T}\right) \cap C^{2}\left(Q_{T}\right), u_{t t x x} \in C\left(Q_{T}\right)\right\} .
$$

Теорема 1. Пусть $a, b \in C^{1}[0, l], q \in C[0, l]$. Тогда существует не более одного решения $u \in \mathscr{C}\left(Q_{T}\right)$ задачи (1), (2).

Доказательств о. Предположим, что существует два различных решения задачи $(1),(2), u_{1}(x, t)$ и $u_{2}(x, t)$. Тогда, в силу линейности задачи, их разность $u=u_{1}-u_{2}$ является решением однородной задачи, соответствующей (1), (2). Покажем, что её решение тривиально. Предварительно заметим, что из физического смысла коэффициентов уравнения и краевых условий функции $a, b, q$ положительны всюду в $Q_{T}$, а $M_{i}, K_{i}$ неотрицательны.

Умножив равенство (1) на $u_{t}$ и проинтегрировав по области $Q_{\tau}$, где $\tau \in[0, T]$ и произвольно, после несложных преобразований получим

$$
\begin{aligned}
& \int_{0}^{l}\left(q u_{t}^{2}(x, \tau)+a u_{x}^{2}(x, \tau)+b u_{x t}^{2}(x, \tau)\right) d x+ \\
& \quad+K_{1} u^{2}(0, \tau)+M_{1} u_{t}^{2}(0, \tau)+K_{2} u^{2}(l, \tau)+M_{2} u_{t}^{2}(l, \tau)=0
\end{aligned}
$$

откуда в силу произвольности $\tau$ сразу вытекает справедливость утверждения теоремы.

Доказательство существования решения проведём для случая постоянных коэффициентов.

Tеорема 2. Пусть $\varphi \in C^{2}[0, l], \varphi(0)=\varphi(l)=\varphi^{\prime \prime}(0)=\varphi^{\prime \prime}(l)=0$, uмеem кусочно непрерывную производную третъего порядка в $(0, l), \psi \in C^{1}[0, l]$, $\psi(0)=\psi(l)=0$ и имеет кусочно непрерывную производную второго порядка в $(0, l), f \in C\left(\bar{Q}_{T}\right)$, тогда решение задачи $(1),(2)$ существует и может быть получено в виде суммы ряда по собственным функииям.

Доказ а mельств о. Будем, как обычно, искать решение задачи в виде суммы

$$
\tilde{u}=u+v
$$


где первое слагаемое - решение поставленной задачи для однородного уравнения, соответствующего (1), второе - решение уравнения (1), удовлетворяющее нулевым начальным и граничным условиям. Воспользуемся результатами проведённых в предыдущем пункте исследований и запишем общее решение уравнения (3):

$$
X(x)=C_{1} \cos \lambda \sqrt{\frac{q}{a-\lambda^{2} b}} x+C_{2} \sin \lambda \sqrt{\frac{q}{a-\lambda^{2} b}} x .
$$

Применив краевые условия (4), приходим к системе уравнений относительно $C_{i}$ :

$$
\begin{aligned}
& \left(a-\lambda^{2} b\right) c_{2}-\left(K_{1}-\lambda^{2} M_{1}\right) c_{1}=0 \\
& \left(-\lambda\left(a-\lambda^{2} b\right) \sin \lambda \sqrt{\frac{q}{a-\lambda^{2} b}} l+\left(K_{2}-\lambda^{2} M_{2}\right) \cos \lambda \sqrt{\frac{q}{a-\lambda^{2} b}} l\right) c_{1}+ \\
& +\left(\lambda\left(a-\lambda^{2} b\right) \sqrt{\frac{q}{a-\lambda^{2} b}} l \cos \lambda \sqrt{\frac{q}{a-\lambda^{2} b}} l+\left(K_{2}-\lambda^{2} M_{2}\right) \sin \lambda \sqrt{\frac{q}{a-\lambda^{2} b}} l\right) c_{2}=0 .
\end{aligned}
$$

Приравнивая нулю ее определитель, получаем спектральное уравнение

$$
\operatorname{ctg} \lambda \sqrt{\frac{q}{a-\lambda^{2} b}} l=\frac{\left(a-\lambda^{2} b\right) \lambda^{2} q-\left(K_{1}-\lambda^{2} M_{1}\right)\left(K_{2}-\lambda^{2} M_{2}\right)}{\lambda \sqrt{q\left(a-\lambda^{2} b\right)}\left(K_{1}+K_{2}-\lambda^{2}\left(M_{1}+M_{2}\right)\right)} .
$$

Выясним, имеет ли это трансцендентное уравнение решения. Для этого рассмотрим функции, стоящие в левой и правой его частях, и исследуем их поведение. Не слишком ограничивая общность, положим

$$
M_{1}=M_{2}=M, \quad K_{1}=K_{2}=K,
$$

что позволит слегка упростить необходимые вычисления. Уравнение (8) принимает вид

$$
\operatorname{ctg} \lambda \sqrt{\frac{q}{a-\lambda^{2} b}} l=\frac{\lambda \sqrt{a-\lambda^{2} b} \sqrt{q}}{2\left(K-\lambda^{2} M\right)}-\frac{K-\lambda^{2} M}{2 \lambda \sqrt{q} \sqrt{a-\lambda^{2} b}} .
$$

Обозначим

$$
\mu=\frac{\lambda \sqrt{q} l}{\sqrt{a-\lambda^{2} b}}
$$

и запишем в новых обозначениях спектральное уравнение:

$$
\operatorname{ctg} \mu=\frac{a q l \mu}{2 K q l^{2}+2 \mu^{2}(K b-a M)}-\frac{K q l^{2}+\mu^{2}(K b-a M)}{2 \mu a q l} .
$$

Анализ функций левой и правой частей последнего уравнения позволяет утверждать, что существует счётное множество его корней и, стало быть, счётное множество собственных функций задачи Штурма-Лиувилля (3), (4), которые с учетом соотношения, полученного из системы относительно $c_{i}$, можно выписать

$$
X_{n}(x)=\cos \lambda_{n} \sqrt{\frac{q}{a-\lambda_{n}^{2} b}} x+\frac{K-\lambda_{n}^{2} M}{\lambda_{n} \sqrt{a-\lambda_{n}^{2} b} \sqrt{q}} \sin \lambda_{n} \sqrt{\frac{q}{a-\lambda_{n}^{2} b}} x .
$$


Теперь перейдём к отысканию решения, удовлетворяющего и начальным условиям. Решение задачи для однородного уравнения мы теперь легко найдём в виде ряда

$$
u(x, t)=\sum_{n=1}^{\infty} T_{n}(t) X_{n}(x)
$$

коэффициенты которого можно найти из начальных данных, пользуясь свойством ортогональности функций $X_{n}(x)$, норма которых может быть получена из соотношения (7):

$$
\|X\|^{2}=\int_{0}^{l}\left(q X_{n}^{2}+b X_{n}^{\prime 2}\right) d x+M_{1} X_{n}^{2}(0)+M_{2} X_{n}^{2}(l) .
$$

Процесс нахождения функции $v(x, t)$ также является, по существу, стандартным, но мы всё же заметим, что, отыскивая решение в традиционном виде

$$
v(x, t)=\sum_{n=1}^{\infty} \tilde{T}_{n}(t) X_{n}(x)
$$

мы получаем два уравнения. Действительно, учитывая вид собственных функций, уточним структуру ряда, в виде которого мы ищем решение:

$$
\begin{aligned}
v(x, t)=\sum_{n=1}^{\infty}\left(V_{n}(t) \cos \lambda \sqrt{\frac{q}{a-\lambda_{n}^{2} b}} x+\right. & \\
& \left.\quad+W_{n}(t) \frac{K-\lambda_{n}^{2} M}{\lambda_{n} \sqrt{a-\lambda_{n}^{2} b} \sqrt{q}} \sin \lambda_{n} \sqrt{\frac{q}{a-\lambda_{n}^{2} b}} x\right) .
\end{aligned}
$$

Для выполнения нулевых начальных условий $v(x, 0)=v_{t}(x, 0)=0$ потребуем, чтобы $V_{n}(0)=V_{n}^{\prime}(0)=0, W_{n}(0)=W_{n}^{\prime}(0)=0$. Разложив $f(x, t)$ в ряд Фурье по собственным функциям $X_{n}(x)$, найдём коэффициенты $f_{n}(t)$ и $g_{n}(t)$. Подставив (9) в уравнение (1), записанное относительно $v(x, t)$, после ряда преобразований получим уравнения для отыскания $V_{n}(t)$ и $W_{n}(t)$ :

$$
\begin{gathered}
V_{n}^{\prime \prime}(t)+\lambda_{n}^{2} V_{n}(t)=\frac{a-\lambda_{n}^{2} b}{a q} f_{n}(t), \\
W_{n}^{\prime \prime}(t)+\lambda_{n}^{2} W_{n}(t)=\lambda_{n} \frac{\left(a-\lambda_{n}^{2} b\right) \sqrt{a-\lambda_{n}^{2} b}}{a \sqrt{q}\left(K-\lambda_{n}^{2} M\right)} g_{n}(t) .
\end{gathered}
$$

Учитывая начальные условия $V_{n}(0)=V_{n}^{\prime}(0)=0, W_{n}(0)=W_{n}^{\prime}(0)=0$, приходим к задачам Коши относительно каждой из функций $V_{n}(t)$ и $W_{n}(t)$, однозначная разрешимость которых гарантирована условиями теоремы. Свойства начальных данных, сформулированные в теореме, не оставляют сомнений в сходимости всех рядов, возникших в ходе наших исследований и, стало быть, в существовании решения поставленной задачи.

Заключение. Доказано существование ортогональной с нагрузкой системы собственных функций исследуемой задачи и получено их представление. 
Установленные свойства собственных функций позволили доказать существование единственного решения поставленной задачи. Отметим, что полученные в статье результаты могут быть использованы как для дальнейших теоретических исследований задач с динамическими граничными условиями, так и для практических целей, а именно для расчёта продольных колебаний широкого круга технических объектов.

\section{ORCID}

Александр Борисович Бейлин: http://orcid.org/0000-0002-4042-2860

\section{БИБЛИОГРАФИЧЕСКИЙ СПИСОК}

1. Нерубай М. С., Штриков Б. Л., Калашников В. В. Ультразвуковая механическая обработка и сборка. Самара: Самарское книжное изд-во, 1995. 191 с.

2. Хмелёв В. Н., Барсуков Р. В., Цыганок С. Н. Ультразвуковая размерная обработка материалов. Барнаул: Алтайский технический ун-т им. И.И. Ползунова, 1997. 120 с.

3. Кумабэ Д. Вибрачионное резание. М.: Машиностроение, 1985. 424 с.

4. Тихонов А. Н., Самарский А. А. Уравнения математической физики. М.: Наука, 2004. $798 \mathrm{c.}$

5. Стретт Дж. В. Теория звука. Т. 1. М.: ГИТТЛ, 1955. 504 с.

6. Rao J. S. Advanced Theory of Vibration: Nonlinear Vibration and One Dimensional Structures. New York: John Wiley \& Sons, Inc., 1992. 431 pp.

7. Федотов И. А., Полянин А. Д., Шаталов М. Ю. Теория свободных и вынужденных колебаний твердого стержня, основанная на модели Рэлея // ДАН, 2007. Т. 417, № 1. C. $56-61$.

8. Bažant Z., Jirásek M. Nonlocal Integral Formulations of Plasticity and Damage: Survey of Progress // J. Eng. Mech., 2002. vol.128, no.11. pp. 1119-1149. doi: 10.1061/(ASCE) 0733-9399(2002) 128:11(1119).

9. Бейлин А. Б., Пулькина Л. С. Задача о продольных колебаниях стержня с динамическими граничными условиями // Вестн. СамГУ. Естественнонаучн. сер., 2014. № 3(114). C. 9-19.

10. Корпусов М. О. Разрушение в неклассических волновых уравнениях. М.: URSS, 2010. 237 c.

Поступила в редакцию 10/II/2016;

в окончательном варианте - 18/V/2016;

принята в печать $-27 / \mathrm{V} / 2016$. 
Vestn. Samar. Gos. Techn. Un-ta. Ser. Fiz.-mat. nauki

[J. Samara State Tech. Univ., Ser. Phys. \& Math. Sci.], 2016, vol. 20, no. 2, pp. 249-258

ISSN: 2310-7081 (online), 1991-8615 (print)

doi: http://dx.doi.org/10.14498/vsgtu1474

MSC: 35L35, 35Q74

\title{
A PROBLEM ON LONGITUDINAL VIBRATION OF A BAR WITH ELASTIC FIXING
}

\section{A.B. Beylin}

Samara State Technical University,

244, Molodogvardeyskaya st., Samara, 443100, Russian Federation.

\begin{abstract}
In this paper, we study longitudinal vibration in a thick short bar fixed by point forces and springs. For mathematical model we consider a boundary value problem with dynamical boundary conditions for a forth order partial differential equation. The choice of this model depends on a necessity to take into account the result of a transverse strain. It was shown by Rayleigh that neglect of a transverse strain leads to an error. This is confirmed by modern nonlocal theory of vibration. We prove existence of orthogonal with load eigenfunctions and derive representation of them. Established properties of eigenfunctions make possible using the separation of variables method and finding a unique solution of the problem.
\end{abstract}

Keywords: dynamic boundary conditions, longitudinal vibration, loaded orthogonality, Rayleigh's model.

\section{ORCID}

Alexander B. Beylin: http://orcid.org/0000-0002-4042-2860

\section{REFERENCES}

1. Nerubai M. S., Shtrikov B. L., Kalashnikov V. V. Ul'trazvukovaia mekhanicheskaia obrabotka $i$ sborka [The ultrasonic machining and assembly]. Samara, Samara Book Publ., 1995, 191 pp. (In Russian)

2. Khmelev V. N., Barsukov R. V., Tsyganok S. N. Ul'trazvukovaia razmernaia obrabotka materialov [The ultrasonic dimensional processing of materials]. Barnaul, 1997, 120 pp. (In Russian)

3. Kumabe J. Vibration Cutting. Tokyo, Jikkyou Publishing Co., Ltd., 1979 (In Japanese).

4. Tikhonov A. N., Samarsky A. A. Uravneniia matematicheskoi fiziki [Equations of Mathematical Physics]. Moscow, Nauka, 2004, 798 pp. (In Russian)

5. Strutt J. W. The theory of sound, vol. 1. London, Macmillan and Co., 1945, xi+326 pp.

6. Rao J. S. Advanced Theory of Vibration: Nonlinear Vibration and One Dimensional Structures. New York, John Wiley \& Sons, Inc., 1992, 431 pp.

(C) 2016 Samara State Technical University.

\section{Please cite this article in press as:}

Beylin A.B. A problem on longitudinal vibration of a bar with elastic fixing, Vestn. Samar. Gos. Tekhn. Univ., Ser. Fiz.-Mat. Nauki [J. Samara State Tech. Univ., Ser. Phys. \& Math. Sci.], 2016, vol. 20, no. 2, pp. 249-258. doi: 10.14498/vsgtu1474. (In Russian)

Author Details:

Alexander B. Beylin (Cand. Techn. Sci.; abeilin@mail.ru), Associate Professor, Dept. of Automation Machine Tools and Tooling Systems. 
7. Fedotov I. A., Polyanin A. D., Shatalov M. Yu. Theory of free and forced vibrations of a rigid rod based on the Rayleigh model, Dokl. Phys., 2007, vol.52, no. 11, pp. 607-612. doi: 10.1134/S1028335807110080.

8. Bažant Z., Jirásek M. Nonlocal Integral Formulations of Plasticity and Damage: Survey of Progress, J. Eng. Mech., 2002, vol. 128, no.11, pp. 1119-1149. doi: 10.1061/(ASCE) 0733-9399 (2002) 128:11(1119).

9. Beylin A. B., Pulkina L. S. A promlem on longitudinal vibrations of a rod with dynamic boundary conditions, Vestnik SamGU. Estestvenno-Nauchnaya Ser., 2014, no. 3(114), pp. 919 (In Russian).

10. Korpusov M. O. Razrushenie v neklassicheskikh volnovykh uravneniiakh [Blow-up in nonclassical wave equations]. Moscow, URSS, 2010, 237 pp. (In Russian)

Received 10/II/2016;

received in revised form $18 / \mathrm{V} / 2016$;

accepted $27 / \mathrm{V} / 2016$. 